\section{Desarrollo cerebral y aprendizaje en adolescentes: Importancia de la actividad física}

\section{Brain development and learning in adolescents: Importance of physical activity}

\section{Señor Editor:}

La adolescencia constituye una gran ventana de plasticidad neuronal, hecho relevante para la biomedicina y la enseñanza. Un interesante artículo en la Revista Médica de Chile ${ }^{1}$ ha enfatizado la importancia de establecer mayor colaboración entre salud y educación, destacando entre los puentes colaborativos a la neuroeducación, disciplina relativamente reciente del estudio del proceso de enseñanza-aprendizaje, que vincula neurociencias con ciencias de la educación ${ }^{1}$. Es conocido que el ejercicio físico se asocia a múltiples beneficios para la salud, pero ¿la actividad física tendrá un impacto sobre la plasticidad cerebral y, por tanto, sobre el aprendizaje de los adolescentes? Una pregunta bastante ambiciosa, pero recientes avances ${ }^{2}$ revelan que la actividad física, además de mejorar la motricidad, activa mecanismos de plasticidad neuronal ${ }^{2}$, favoreciendo el aprendizaje significativo y el almacenamiento de memoria ${ }^{2}$. Considerando lo anterior, esta Carta al Editor desea destacar la importancia que posee la actividad física para el desarrollo cerebral y el aprendizaje en los adolescentes.
El desarrollo cerebral es un proceso continuo para el cual es importante la plasticidad neuronal ${ }^{3}$. Diversos son los compuestos que modulan este proceso y que ejercen gran influencia sobre el sistema nervioso central (SNC), destacando entre ellos: neurotransmisores, opioides, drogas y especialmente hormonas. Si bien el impacto del entrenamiento físico sobre el control neuroendocrino del eje hipotalámico-hipofisario-glandular en niños en desarrollo es complejo ${ }^{4}$, el aumento de secreción adenohipofisaria de hormona del crecimiento $(\mathrm{GH})$ en respuesta al ejercicio depende del estado puberal ya que niños en etapas puberales más avanzadas responden con mayores peaks de GH en comparación con aquellos en etapas más tempranas ${ }^{4}$. Además, el ejercicio físico en niños y adolescentes modularía la secreción de hormonas esteroidales ${ }^{4}$, hecho relevante pues actualmente se conoce que éstas (e.g., hormonas sexuales y glucocorticoides) actúan mediante efectos activacionales y efectos organizacionales: los primeros modifican la actividad neuronal que favorece un comportamiento dado en un contexto específico, no son permanentes y aparecen debido a la acción de un estímulo ${ }^{3}$; los segundos determinan la estructura del SNC durante su desarrollo, permaneciendo en el tiempo después de la exposición al compuesto y permitiendo la generación de respuestas de activación al exponerse a dichos estímulos en la adultez ${ }^{3}$. Estos efectos organizacionales se agrupan en mielinización, poda neuronal, apoptosis, remodelación dendrítica y cambios epigenéticos (Figura 1).

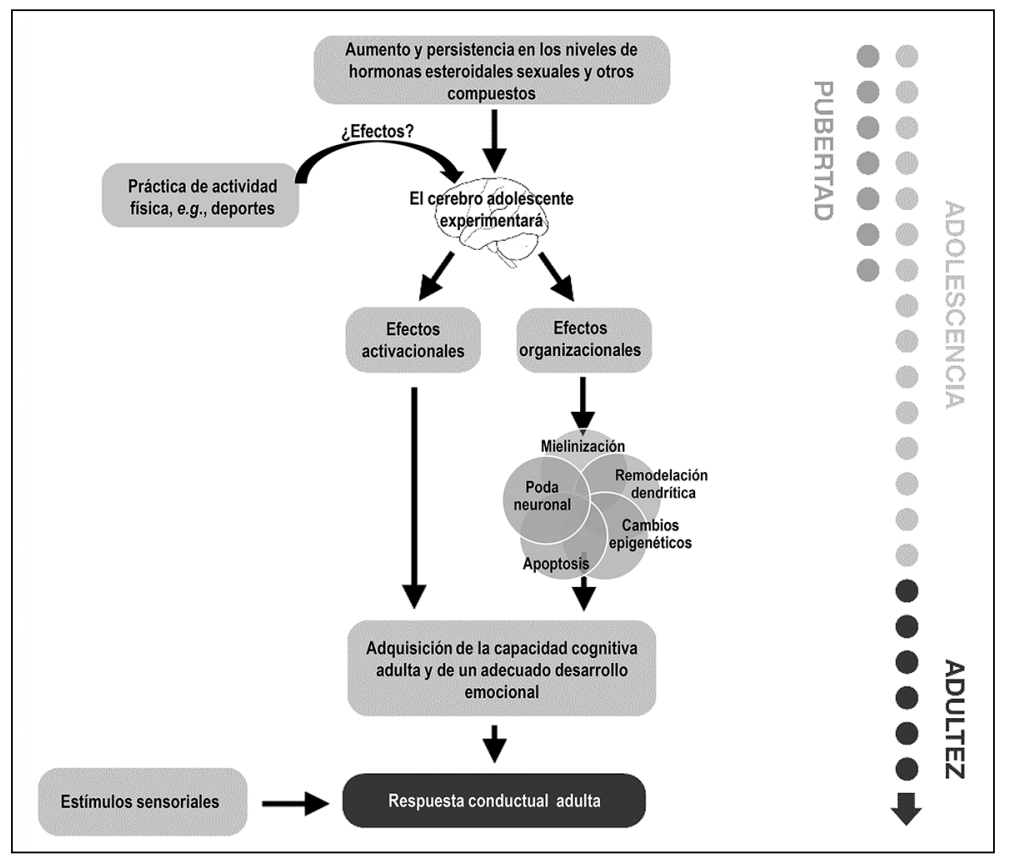

Figura 1. Efectos organizacionales y activacionales de hormonas en el cerebro adolescente. El ejercicio físico modularía los efectos de hormonas y otros compuestos. 
En consecuencia, las hormonas esteroidales -que aumentan durante pubertad y adolescencia y cuya secreción es modulable por el ejercicio físico- pueden desencadenar cambios estructurales permanentes en el cerebro ${ }^{3}$, lo cual afecta el proceso de aprendizaje en adolescentes.

Los profesores de educación física saben por su labor formativa diaria que la práctica de ejercicio físico permite a adolescentes el desarrollo de habilidades tales como liderazgo, trabajo en equipo, capacidad de razonamiento lógico, control de ansiedad y autocuidado personal. Éstas son importantes cotidianamente e impactan positivamente en el proceso de aprendizaje y el desarrollo integral de los educandos. En general, el ejercicio físico desencadena efectos placenteros y motivacionales, lo que puede incentivar para seguir ejercitándose. Estos efectos se atribuyen a la secreción durante el ejercicio de endocannabinoides y opioides endógenos (encefalinas, endorfinas y dinorfinas), que poseen diversas funciones en el SNC. Un reciente estudio en Nature Communications ${ }^{5}$ demostró que en humanos estos opioides regulan el aprendizaje de amenazas sociales. Esto apoya el hecho de que el ejercicio no solamente generaría beneficios en la salud física, sino también en la salud mental.

Desde la Antigüedad escuchamos mens sana in corpore sano y suena lógico, nadie lo refuta. Este adagio latino adquiere creciente validez a la luz de recientes investigaciones en neurociencias ${ }^{2,3,5}$ y fisiología deportiva ${ }^{4}$. Sin embargo, el gran costo económico de la inactividad física en Chile ${ }^{6}$ unido al sobrepeso y la obesidad escolar recuerda otro adagio: per aspera ad astra, es decir, la necesidad de esforzarse para lograr triunfos en esta área. Es esencial, entonces, desarrollar políticas públicas que fomenten el ejercicio físico regular ${ }^{6}$, que incrementen las horas de actividad física en los establecimientos educacionales ${ }^{6}$, que prevengan el consumo de drogas y, en general, que promuevan el autocuidado y los hábitos de vida saludable entre escolares. Esto colaborará en el desafío de lograr que nuestros adolescentes tengan mejor salud, bienestar y condiciones propicias para el aprendizaje.

Agradecimientos: Al Dr. Juan Pablo del Río, médico cirujano (Universidad de los Andes, Chile) por sus comentarios. Al Proyecto UBO/VRIP 1803.
Manuel E. Cortés-Cortés ${ }^{1,2}$, Andrea Alfaro Silva, Valeska Martínez ${ }^{5,6}$, Bianca C. Veloso ${ }^{7}$

${ }^{1}$ Biólogo, Profesor de Química y Biología, Ingeniero en Prevención de Riesgos (registrado en SEREMI-RM),

M.B.A., M.Sc., Ph.D.

${ }^{2} J e f e$ de Departamento de Ciencias Químicas y Biológicas, Facultad de Salud, Universidad Bernardo O'Higgins $(U B O)$.

${ }^{3}$ Profesora de Biología, Magister en Educación (M.Ed.) en Salud y Bienestar Humano.

${ }^{4}$ Coordinadora de Unidad de Género y Sexualidad, Liceo Experimental Manuel de Salas, Universidad de Chile.

${ }^{5}$ Profesora de Educación Física, Deportes y Recreación. ${ }^{6}$ Tesista M.Ed., UBO.

${ }^{7}$ Estudiante de Educación Física, UBO, Santiago, Chile.

\section{Referencias}

1. Marqués ML, Osses S. Neurociencia y educación: una nueva dimensión en el proceso educativo. Rev Med Chile 2014; 142 (6): 805-6.

2. Navarro B, Osses S. Neurociencias y actividad física: una nueva perspectiva en el contexto educativo. Rev Med Chile 2015; 143 (7): 950-1.

3. Vigil P, del Río JP, Carrera B, Aránguiz FC, Rioseco H, Cortés ME. Influence of sex steroid hormones on the adolescent brain and behavior: An update. Linacre Q 2016; 83 (3): 30829.

4. Richmond E, Rogol AD. Endocrine responses to exercise in the developing child and adolescent. Front Horm Res 2016; 47: 58-67.

5. Haaker J, Yi J, Petrovic P, Olsson A. Endogenous opioids regulate social threat learning in humans. Nat Commun 2017; 8: 15495.

6. Celis-Morales C, Salas C, Martínez MA, Leiva AM, Garrido-Méndez A, Díaz-Martínez X. Costo económico asociado a inactividad física en Chile. Rev Med Chile 2017; 145 (8): 1091-2.

Correspondencia a:

Dr. Manuel E. Cortés-Cortés

Departamento de Ciencias Químicas y Biológicas, Facultad de

Salud, Universidad Bernardo O'Higgins, General Gana 1702,

Santiago, Chile.

cortesmanuel@docente.ubo.cl 